\title{
Theory of gear adaptive transmission
}

\author{
Konstantin Ivanov ${ }^{1,2, *}$ \\ ${ }^{1}$ Chair "Control Systems for Aerospace Engineering", Almaty University of Power Engineering and Telecommunications, Almaty, Kazakhstan \\ ${ }^{2}$ Laboratory of Adaptive Mechanisms, Institute of Mechanics and Machine Science MON of Republic Kazakhstan, Almaty, Kazakhstan
}

\section{Email address:}

ivanovgreek@mail.ru

\section{To cite this article:}

Konstantin Ivanov. Theory of Gear Adaptive Transmission. American Journal of Mechanics and Applications. Special Issue: Adaptive Transmissions. Vol. 2, No. 6-1, 2014, pp. 5-12. doi: 10.11648/j.ajma.s.2014020601.12

\begin{abstract}
Gear adaptive transmission represents itself as the gear variator with the variable transfer ratio. Unsuccessful attempts of creation of a gear variator were undertaken repeatedly. The main problem is - maintenance of constant gearing of cogwheels of a variator. The decision of this problem is based on use of science discovery in the kinematic chain with two degrees of freedom. The gear variator in the form of the closed differential mechanism has definability and allows changing the transfer ratio in the set range. Thus external variable technological loading changes the transfer ratio itself. The adaptive to variable loading gear variator works without the control mechanism. The gear variator opens essentially new concept of creation of adaptive techniques for machines with variable technological resistance (for example, adaptive gearboxes). The theory of a gear variator is based on a discovery «Effect of force adaptation in mechanics», published in leading editions of world press. According to this discovery the kinematic chain with two degrees of freedom, containing the mobile closed contour, becomes the definable mechanism at presence only one input link. Such mechanism gets property independently to change speed of motion of an output link depending on variable loading. The discovery is based on mechanics laws. In work the bases of the theory of a gear adaptive variator are stated.
\end{abstract}

Keywords: Gear Variator, Differential Mechanism, Force Adaptation

\section{Introduction}

The variator is a mechanism for stepless regulating of the transfer ratio. At present there are only frictional variators.

The gear variator is a wheelwork with constant cogging of toothed wheels and with the variable transfer ratio. The gear variator is dream of the designers creating drives of cars with variable technological resistance (for example, automobile gear boxes). The gear variator unlike a frictional variator can procure reliable transfer of force and high efficiency.

Earlier attempts of creation of adaptive gear variators in the form of mechanisms with two degrees of freedom in which speed of rotate of the output shaft changes as a function of loadings were undertaken [1, 2 and 3]. Ivanov [1] used a wheelwork as the kinematic chain between the mobile stator and rotor of electric motor, Crockett [2] used a gear variator together with the fluid converter, and Harris [3] used a brake for management of the mechanism. Patents of originators did not contain authentic theoretical dependences for calculation of mechanisms.

The theoretical substantiation for creation of adaptive gear variators has been developed in works of Ivanov K.S. [4, 5 and 6]. It has been proved, that the kinematic chain with two degrees of freedom, containing the mobile closed contour, possesses brand new property of force adaptation. The found unknown phenomenon was as the science discovery and has received the name «Effect of force adaptation in mechanics». According to this discovery the kinematic chain with two degrees of freedom and one input, containing the closed contour, independently changes a motion output speed under the acting of a variable loading. Such kinematic chain is an adaptive gear variator with constant cogging of toothed wheels. The gear variator was named as the adaptive mechanism.

The adaptive mechanism allows transferring motion from the propeller of constant power to the actuator with a speed, inversely proportional to technological loading. Discovery of effect of force adaptation has allowed finding brand new regularity of interacting of power and kinematic parameters of the kinematic chain with two degrees of freedom. This regularity defines kinematics and dynamics of the adaptive mechanism and allows performing the analysis and synthesis for it.

Essentially new feature of an adaptive gear variator consists that it unlike a frictional variator works independently without use of any control system. Variable external loading operates a variator delivery speed. It would be possible to name such 
control mode as the transfer ratio self-adjustment, and it is possible to consider the adaptive mechanism as the mechanism self-adjusted to a variable load.

Regularity of force adaptation has allowed creating brand new schemes of adaptive variators. In Ivanov's patents [7, 8 and 9] gear variators with a backing, with a high range of transfer ratios, with the use of inertia properties are presented.

The purpose of the present work consists in presenting the short theory of a gear variator on the basis of of mechanics laws and the usual techniques of the structural, kinematic and dynamic analysis of the mechanisms.

\section{Description of Gear Adaptive Mechanism}

In present time the scientific researches of so-called adaptive mechanisms which procure self-adjustment to external loading are executed. The adaptive connecting gear provides transfer of motion from the propeller of constant power on the tool with a speed, inversely proportional loading. The adaptive mechanism possesses property of mechanical adaptation. Mechanical adaptation is an ability of the mechanism independently without any control system to be accommodated for variable technological loading. The functional essence of the adaptive mechanism is maintenance of the optimum variable transfer ratio at constant power of an engine. This function essentially differs from function of the gear box. The gear box has a few drive stages. At each stage it is necessary to change power of an engine for achievement of optimum result of an energy transfer. Unlike the step gear box the adaptive connecting gear provides brand new phenomenon in technique - self-adjustment to variable technological loading at constant power of an engine without control system use. The adaptive connecting gear essentially differs from a gear box by absence of a control system. Thus, it is possible to consider the adaptive mechanism as the mechanism self-adjusted to a variable load.

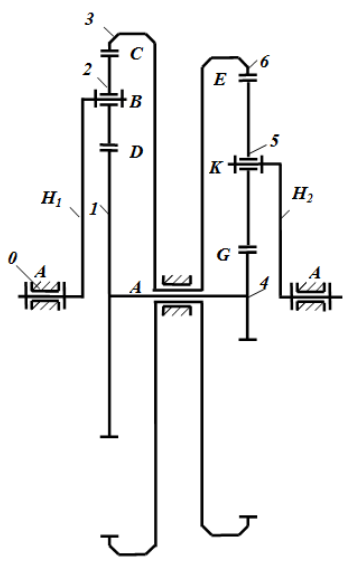

Fig. 1. Gear variator

The adaptive wheelwork (gear variator) looks like the closed gear differential with two degrees of freedom (fig. 1). It contains a rack 0 , the carrier $H_{1}$, closed contour, containing toothed wheels 1-2-3-6-5-4 and the carrier $\mathrm{H}_{2}$. Solar wheels
1, 4 are merged in the block of wheels 1-4. Epicycle wheels 3, 6 are merged in the block of wheels 3-6. The kinematic chain has two degrees of freedom.

Features of the kinematic chain:

1) Chain has two external links (carriers $H_{1}$ and $H_{2}$ ) which are connected by structural group 1-2-3-6-5-4 with zero mobility. This structural group represents mobile closed contour.

2) Instant centers of speeds of the central toothed wheels are coincide (they are placed on the central axis of gear differential).

\subsection{Work of a Gear Variator}

The gear variator can work in a regime with two degrees of freedom and in a regime with one degree of freedom.

Motion of the mechanism with two degrees of freedom occurs in operating conditions of motion with self-regulation.

Motion with one degree of freedom occurs in two cases:

1)At variator start-up when the output carrier is shut down.

2)At an overloading when the moment of resistance on the output carrier exceeds the maximum value that also leads to a stop of the output carrier.

At first let's consider the variator start-up.

The variator placed between the propeller and the tool of the car, admits start-up with gradual increase in a moment of resistance (with clutch use) and with direct affecting on the tool (without clutch use).

The clutch should be placed after the variator output shaft. The start-up beginning occurs at the disconnected tool. The mechanism of a variator in the absence of output loading passes in motion with one degree of freedom at the rotating output carrier. Angular speeds of all links are equal and equal to input angular speed. The contour from toothed wheels is rotating as a single whole in the absence of relative motion of wheels in a contour. The moment of resistance on the output carrier can occur at act of the inertia forces, or in the presence of an internal friction. The greatest possible moment of resistance on the output carrier is equal to the input driving moment.

After the joint of a variator with the tool (by means of the clutch) the variator mechanism passes in motion operating conditions. Motion with two degrees of freedom in the presence of relative motion of toothed wheels in the closed contour begins. The increase in a output moment of resistance leads to decrease of output angular speed and to start-up from a place. After start-up the operating conditions of motion begin with a place with presence of effect of force adaptation.

Start-up of a variator in the absence of the clutch occurs at the motionless output carrier. Start-up from a place of the output carrier can occur in the presence of an internal moment of resistance in the closed contour which can be presented in the form of some resulted moment of resistance $M_{R 5}$ on the output satellite 5. Moment of resistance $M_{R 5}$ occurs as the inertia moment or as the moment of a frictional force. In a break-away torque from a place the greatest possible moment of resistance on the output carrier is equal to the input driving 
moment. Therefore start-up should occur in a regime of increase in power of an engine. After start-up the operating conditions of motion begin with a place of the output carrier with presence of effect of force adaptation.

Let's consider variator work at an overloading.

When the moment of resistance on the output carrier exceeds the maximum value, there is a stop of the output carrier. The propeller continues to work at the motionless tool. At the working propeller and the shut down tool it is possible to name an operating mode стоповым a motion regime. Possibility of transition of a variator in the stop motion regime has the important practical value. Stop motion regime allows preventing a mechanism exit out of operation at overloading.

After elimination of an overloading the mechanism will continue work in motion operating conditions.

\section{Structure of Adaptive Wheelwork}

The structure of an adaptive wheelwork basic differs from structure of the multistage connecting gear. The wheelwork with two degrees of freedom has two external links (carriers $H_{1}$ and $H_{2}$ ) and the structural group of Assur with zero mobility placed between them. This structural group represents a closed contour which contains four toothed links 1-2-3-6-5-4.

Number of a degrees of freedom of the kinematic chain is defined by the Tchebyshev formula

$$
W=3 n-2 p_{5}-p_{4}=3 \cdot 6-2 \cdot 6-4=2 .
$$

As it has been noted above, the gear variator can work in a regime with two degrees of freedom and in a regime with one degree of freedom depending on magnitude of output loading.

\section{Kinematic Analysis of Gear Variator}

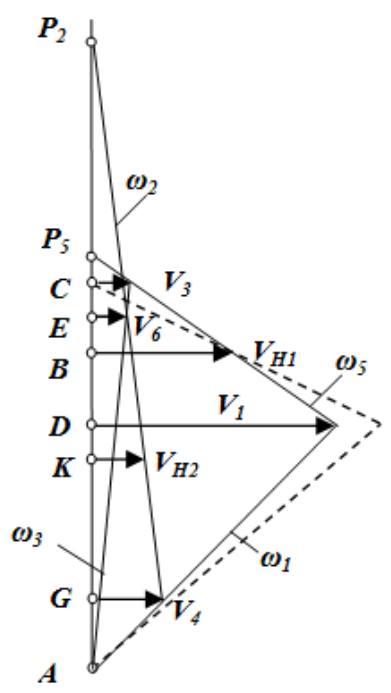

Fig. 2. Picture of speeds of the adaptive mechanism

The gear variator represents the kinematic chain with two degrees of freedom. Therefore the kinematic analysis of a variator consists in definition of speeds of all points of the mechanism on the set speeds of two external links. It is convenient to make the kinematic analysis of a variator by means of a picture of speeds (fig. 2).

In a picture of speeds linear speeds $V_{i}$ points of the mechanism in the form of horizontal lines and angular speeds of links $\omega_{i}$ - in the form of inclined lines are presented.

Angular speeds $\omega_{1}=\omega_{4}, \omega_{3}=\omega_{6}$ intermediate links 1-4 and 3-6 are defined in known angular speeds of external links, водил $\omega_{H 1}, \omega_{H 2}$ and transfer ratios at the shut down carriers.

Transfer ratios of links of transfer we will define through numbers of teeth of wheels $z_{i} i=1,2, \ldots 6$.

The interconnection of angular speeds of the mechanism is defined by formulas

$$
\begin{aligned}
& \frac{\omega_{1}-\omega_{H 1}}{\omega_{3}-\omega_{H 1}}=u_{13}^{(H 1)} \\
& \frac{\omega_{1}-\omega_{H 2}}{\omega_{3}-\omega_{H 2}}=u_{46}^{(H 2)}
\end{aligned}
$$

Where $u_{13}^{(H 1)}=-z_{3} / z_{1}, u_{46}^{(H 2)}=-z_{6} / z_{4}$.

From (1)

$$
\omega_{1}=u_{13}^{(H 1)}\left(\omega_{3}-\omega_{H 1}\right)+\omega_{H 1}
$$

From (2)

$$
\omega_{1}=u_{46}^{(H 2)}\left(\omega_{3}-\omega_{H 2}\right)+\omega_{H 2}
$$

Let's subtract (4) of (3), we will receive

$$
\begin{gathered}
u_{13}^{(H 1)}\left(\omega_{3}-\omega_{H 1}\right)+\omega_{H 1}-u_{46}^{(H 2)}\left(\omega_{3}-\omega_{H 2}\right)-\omega_{H 2}=0 . \text { From here } \\
\left(u_{13}^{(H 1)}-u_{46}^{(H 2)}\right) \omega_{3}-u_{13}^{(H 1)} \omega_{H 1}+u_{46}^{(H 2)} \omega_{H 2}=\omega_{H 2}-\omega_{H 1} \text { From here } \\
\omega_{3}=\frac{\omega_{H 2}\left(1-u_{46}^{(H 2)}\right)-\omega_{H 1}\left(1-u_{13}^{(H 1)}\right)}{u_{13}^{(H 1)}-u_{46}^{(H 2)}}
\end{gathered}
$$

Formulas (5) and (4) define sequence of acts by definition angular speeds $\omega_{3}, \omega_{1}$ transfer links.

Angular speed of the satellite 2 is defined from a condition

$$
\frac{\omega_{2}-\omega_{H 1}}{\omega_{3}-\omega_{H 1}}=u_{23}^{(H 1)}
$$

where $u_{23}^{(H 1)}=z_{3} / z_{2}$. From here

$$
\omega_{2}=u_{23}^{(H 1)}\left(\omega_{3}-\omega_{H 1}\right)+\omega_{H 1}
$$

Angular speed of the satellite 5 is defined from a condition

$$
\frac{\omega_{5}-\omega_{H 2}}{\omega_{3}-\omega_{H 2}}=u_{56}^{(H 2)}
$$


Where $u_{56}^{(H 2)}=z_{6} / z_{5}$. From here

$$
\omega_{5}=u_{56}^{(H 2)}\left(\omega_{3}-\omega_{H 2}\right)+\omega_{H 2}
$$

It is necessary to note, that in the absence of mobility in a contour the kinematic chain will move in a condition with one degree of freedom. In this case angular speeds of all links are equal.

Thus, all kinematic and power parameters are defined, and all mechanism has the kinematic and static definability.

\section{Force Analysis of a Gear Variator}

Let's make the power analysis of the kinematic chain (fig. 2) by the standard technique. The problem of the power analysis of the mechanism with two degrees of freedom consists in definition of reactions in kinematic pairs and in definition of the generalized forces on two external links.

Some features of act of forces occur for an considered kinematic chain. We will consider, that on external links the generalized forces - moments $M_{H 1}$ and $M_{H 2}$ on carriers $H_{1}$ and $H_{2}$ are acting. On intermediate structural group of Assur the superposed forces do not act (a gravity of links and an inertial force of links is neglected because of them малости in comparison with forces on external carriers).

The force analysis should be begun with consideration of structural group 1-2-3-6-5-4 in the form of the closed contour consisting of toothed wheels. The structural group contains the block of solar wheels 1-4, the satellite 2, the block epicycle wheels 3-6 and the satellite 5. Such structural group was never considered earlier in the power analysis of mechanisms. However for this structural group it is possible to make conditions of statics balance which define interconnection internal and superposed forces. We will consider, that superposed forces for considered structural group is force $F_{H 1}$ transferred from the carrier $H_{1}$ on point $B$, and force $F_{H 2}$ transferred from the carrier $H_{2}$ on point $K$. Internal forces are reactions in kinematic pairs in points $C, E, D, G$.

The first feature of considered structural group consists that all internal forces $R_{32}, R_{65}, R_{12}, R_{45}$ can be expressed on statics conditions through active forces $F_{H 1}$ and $F_{H 2}$.

For links of a contour 2 and 5 we will express reactions in the kinematic парах $D, C, G, E$ through external силы $F_{H 1}, F_{H 2}$, enclosed in точках $B, K$

$$
\begin{aligned}
& R_{12}=R_{32}=0.5 F_{H 1} \\
& R_{45}=R_{65}=0.5 F_{H 2}
\end{aligned}
$$

Here

$$
\begin{aligned}
& F_{H 1}=M_{H 1} / r_{H 1}, R_{12}=M_{12} / r_{1}, R_{32}=M_{32} / r_{3} \\
& F_{H 2}=M_{H 2} / r_{H 2}, R_{45}=M_{45} / r_{4}, R_{65}=M_{65} / r_{6} \\
& M_{H 1}, M_{H 2} \text { - moments on carriers, } \\
& r_{H 1}, r_{H 2} \text { - radiuses of carriers, }
\end{aligned}
$$

$M_{12}, M_{32}$ - moments, created on the satellite by 2 reactions $R_{12}, R_{32}$ co of the party of toothed wheels 1 and 3 ,

$M_{45}, M_{65}$ - moments, created on the satellite by 5 reactions $R_{45}, R_{65}$ co of the party of toothed wheels 4 and 6 , $r_{i}(i=1,2 \ldots 6)$ - radiuses of wheels.

After substitution of values of forces in the equations (10), (11) we will receive formulas for definition of the internal moments through the external moments

$$
\begin{aligned}
& M_{12}=0.5 M_{H 1} r_{1} / r_{H 1} \\
& M_{32}=0.5 M_{H 1} r_{3} / r_{H 1}, \\
& M_{45}=0.5 M_{H 2} r_{4} / r_{H 2} \\
& M_{65}=0.5 M_{H 2} r_{6} / r_{H 2}
\end{aligned}
$$

Let's make for the satellite 2 equilibrium equation in the form of the sum of the moments concerning its instant centre of speeds $P_{2}$

$$
R_{12} \cdot P_{2} D+R_{32} \cdot P_{2} C=F_{H 1} \cdot P_{2} B
$$

Let's increase the equation (16) on $\varphi_{2}\left(\varphi_{2}\right.$ - an angle of turn of a link 2 around pole $P_{2}$ ), we will receive

$$
R_{12} s_{D}+R_{32} s_{C}=F_{H 1} s_{B}
$$

Let's make for the satellite 5 equilibrium equation in the form of the sum of the moments concerning its instant centre of speeds $P_{5}$

$$
R_{45} \cdot P_{5} G+R_{65} \cdot P_{5} E=F_{H 2} \cdot P_{5} K
$$

Let's increase the equation (18) on $\varphi_{5}\left(\varphi_{5}\right.$ - an angle of turn of a link 5 around pole $P_{5}$ ), we will receive

$$
R_{45} s_{G}+R_{65} s_{E}=R_{H 2} s_{K}
$$

Here $S_{B}, S_{C}, S_{D}, S_{E}, S_{G}, s_{K}$ - the valid displacements of points $B, C, D, E, G, K$.

Let's express displacements of points through instant angles of turn of links concerning the central axis of the mechanism and radiuses: $s_{D}=\varphi_{1} r_{1}, s_{C}=\varphi_{3} r_{3}, s_{B}=\varphi_{H 1} r_{H 1}$,

$$
s_{G}=\varphi_{4} r_{4}, s_{E}=\varphi_{6} r_{6}, s_{K}=\varphi_{H 2} r_{H 2} .
$$

$\varphi_{1}, \varphi_{3}, \varphi_{H 1}, \varphi_{4}, \varphi_{6}, \varphi_{H 2}$ - instant angles of turn of toothed wheels and carriers.

Formulas (17), (19) define possibility of transformation of the equations of the moments (16), (18) in equilibrium equations by a principle of possible displacements (17), (19) with use of the valid displacements instead of the possible.

Formulas (17), (19) match to a principle of possible displacements for all kinematic chain as the instant centres of 
speeds of the central toothed wheels 1-4 and 3-6 coincide with the central axis of the mechanism.

Taking into account $\varphi_{1}=\varphi_{4}, \varphi_{3}=\varphi_{6}$ and time we will receive

$$
\begin{gathered}
M_{12} \omega_{1}+M_{32} \omega_{3}=M_{H 1} \omega_{H 1} \\
M_{45} \omega_{1}+M_{65} \omega_{3}=M_{H 2} \omega_{H 2}
\end{gathered}
$$

As satellites 2 and 5 are a part of the mechanism as a whole, we will combine the made expressions. We will receive a condition of interacting of parameters of the kinematic chain as a whole

$$
\begin{gathered}
M_{12} \omega_{1}+M_{32} \omega_{3}+M_{45} \omega_{1}+M_{65} \omega_{3}= \\
=M_{H 1} \omega_{H 1}+M_{H 2} \omega_{H 2}
\end{gathered}
$$

In the left side of an equation (22) the sum of powers (matching to the sum of works) contour internal forces occurs.

In the considered mechanism all internal forces are defined through the known superposed forces, all internal displacements are defined through external displacements. Hence, work (or power) of internal forces on possible internal displacements is defined. Constraints in kinematic pairs are ideal and stationary. Work of superposed forces cannot pass in work of internal forces. Hence, work (power) of internal forces on possible internal displacements is equal to null

$$
M_{12} \omega_{1}+M_{32} \omega_{3}+M_{45} \omega_{1}+M_{65} \omega_{3}=0
$$

The right side of an equation (22) represents the sum of powers (matching to the sum of works) contour superposed forces. At performance of a condition (23) we will receive from the equation (22) condition of balance for superposed forces according to a principle of possible works

$$
M_{H 1} \omega_{H 1}+M_{H 2} \omega_{H 2}=0
$$

The resulted dependences allow defining all power parameters of the kinematic chain that testifies to its static definability.

The made power analysis of the mechanism with two degrees of freedom allows making following conclusions:

1) Equation of the moments can be converted to an equilibrium equation by a principle of possible displacements.

2) Considered kinematic chain in the form of the differential mechanism with the closed contour containing toothed wheels, allows receiving the general equilibrium equation of all chain in the form of the equation of interconnection of power and kinematic parameters by a principle of virtual displacements.

3) It is obviously possible (Eq. 22) to present the general condition of interconnection of power and kinematic parameters in the form of two separate parts for superposed forces and for internal forces.

4) The received condition of balance on a principle of possible displacements for internal forces allows considering the kinematic chain as system with ideal Connections for which the sum of works of internal forces is equal to null (23).

5) From the assumption of ideality of the kinematic chain follows, that the sum of works of superposed forces also is equal to null (Eq. 24) that predetermines a uniform motion of the kinematic chain.

6) Condition of interconnection of superposed forces (Eq. 24) predetermines presence of works with unlike signs on external chain links (carriers $H_{1}$ and $H_{2}$ ). The link with presence of negative work cannot be an input link as force acting on it is not an impellent, and force of resistance.

This main leading-out leads to necessity to consider a considered kinematic chain as a chain containing only one input link.

The resulted conclusion does not change the status of definability of a considered kinematic chain. As the kinematic chain remains definable, it is necessary to consider it as the mechanism with two degrees of freedom, but only with one input. Thus the kinematic definability of a chain remains, as for an output link the generalized co-ordinate (speed) is defined from the equation of interconnection of external parameters (Eq. 24). We will consider as an input link the carrier $H_{1}$. An output link is the carrier $H_{2}$. From the Eq. 24 it follows

$$
\omega_{H 2}=M_{H 1} \omega_{H 1} / M_{H 2}
$$

Thus the external moments remain set, but $M_{H 1}$ - the input driving moment, and $M_{H 2}$ - a output moment of resistance. The Eq. expresses the additional communication imposed by a contour on motion of chain links with two degrees of freedom.

7) Additional constraint (Eq. 25) provides effect of force adaptation to output loading: at the set constant parameters of aerial input $M_{H 1}, \omega_{H 1}$ and set output moment of resistance $M_{H_{2}}$ output angular speed $\omega_{H 2}$ is in return proportional dependence on the variable output moment сопротивления $M_{H 2}$.

Eq. 25 matches to the theorem about the closed contour [4] the closed contour provides force adaptation to a variable load.

8) The condition of interconnection of internal forces (Eq. 23) predetermines presence of works with unlike signs on internal chain links.

From the equation (Eq. 23) we will receive

$$
\left(M_{12}+M_{45}\right) \omega_{1}+\left(M_{32}+M_{65}\right) \omega_{3}=0 .
$$

Taking into account signs on the moments (driving moments $M_{12}, M_{32}$, transferred from the input satellite 2 are positive, the moments of resistance $M_{45}, M_{65}$, transferred from the output satellite 5 , are negative) we will receive

$$
\left(M_{12}-M_{45}\right) \omega_{1}+\left(M_{32}-M_{65}\right) \omega_{3}=0
$$

The equation 26 represents the equation of works (powers) on intermediate links 1-4 and 3-6. The equation 26 means balance presence on intermediate links 1-4 and 3-6 simultaneously. In the mobile closed contour basic new situation occurs: balance in statics separately on each 
intermediate link is absent, but balance of intermediate links simultaneously on the move all contour occurs.

In the closed contour energy circulation occurs.

The equation 26 contains positive and negative members and characterizes balance of powers on intermediate links of a contour. As for the considered scheme, $M_{45}>M_{12}, M_{32}>M_{65}$ To from the equation 26 we will receive

$$
-\left(M_{45}-M_{12}\right) \omega_{1}+\left(M_{32}-M_{65}\right) \omega_{3}=0
$$

From here

$$
\left(M_{45}-M_{12}\right) \omega_{1}=\left(M_{32}-M_{65}\right) \omega_{3}
$$

Or $\left(M_{54}-M_{21}\right) \omega_{1}=\left(M_{23}-M_{56}\right) \omega_{3}$.

Let's mark out $M_{54}-M_{21}=M_{1-4}, M_{23}-M_{56}=M_{3-6}$.

Here $M_{1-4}, M_{3-6}$ the moments on blocks of wheels 1-4 and 3-6. Then we will receive

$$
M_{1-4} \omega_{1}=M_{3-6} \omega_{3}
$$

The equation (29) reflects an analytical form of circulation of energy unknown earlier in a contour during its motion. Thus, it is installed, that the gear variator represents the closed gear differential mechanism with two degrees of freedom and with one input.

\section{Efficiency of Gear Variator}

The adaptive wheelwork has two degrees of freedom. However efficiency of this mechanism basic differs from efficiency of usual mechanisms with two degrees of freedom (a clutch friction coupling, hydrodynamic transfer) in which the friction carries out impellent function. In the gear adaptive mechanism impellent function is not based on friction use. Impellent function is carried out by the closed contour from toothed wheels. An energy loss on a friction in the gear adaptive mechanism is connected only with motion of toothed wheels as in a usual wheelwork and not based on friction use as impellent function. Therefore efficiency of an adaptive wheelwork is considerably above than efficiency of a friction coupling of the clutch or efficiency of hydrodynamic transfer.

Hydrodynamic transfer is transfer with two degrees of freedom. The transfer ratio of hydrodynamic transfer can change from 1 to the maximum value (nearby 2 ) when there is an output shaft stop. At the moment of a stop of the output shaft of efficiency it is equal 0 .

However, in hydrodynamic transfer the output moment is created by an internal hydraulic moment of resistance which depends on a difference of angular speeds of pump and turbine wheels. The kinematic joint of pump and turbine wheels cannot be an ideal kinematic pair. This joint functionally exists for transformation of an internal moment of resistance to an external output moment of resistance. The hydraulic moment of resistance simultaneously defines factor of an energy loss. Therefore efficiency of hydrodynamic transfer linearly decreases depending on loading.

Basic other dependence occurs in mechanical adaptive transfer. This dependence is defined by the formula (25) connecting the external moments and speeds. The internal mechanical moment in the closed contour of mechanical adaptive transfer also occurs. However this moment does not create energy loss, as power (or work) internal forces is equal to null. In mechanical adaptive transfer there is only a redistribution of speeds of motion of links in the closed contour.

Thus, efficiency of mechanical adaptive transfer functionally does not depend on external loading and matches to efficiency of the planet gear. At the moment of qualitative change of structure of the mechanism at a stop of an output link of efficiency gets an instant zero value. However the mechanism passes in a new condition with one degree of freedom, in which efficiency is defined by appropriate amount.

\subsection{Efficiency of the Adaptive Gearing}

Efficiency of the adaptive gearing we will define by formula

$$
\eta=\frac{N_{H 2}}{N_{H 1}}
$$

Where $N_{H_{2}}$ - a useful power on output carrier $\mathrm{H}_{2}$, $N_{H 1}$ - spent power on input carrier $H_{1}$.

The adaptive gearing (fig. 1) represents the gear planetary train which depending on the enclosed loading can move with one or with two degrees of freedom. Efficiency of adaptive transfer depends on a motion regime.

The adaptive gearing moves with one degree of freedom if the output moment of resistance is equal to the input driving moment $M_{H 2}=M_{H 1}$.

In this case the closed contour which is switching on toothed wheels $1,2,3,6,5,4$, moves as a single whole without relative mobility of toothed wheels in a contour. A friction loss occurs only in two rotational kinematic pairs $A$, which are uniting input and output carriers with a rack. Therefore $N_{H 2}=N_{H 1} \eta_{A}^{2}$,

Where $\eta_{A}=0.99$ - efficiency of rotational pair $A$.

Efficiency of all mechanism in a regime of motion with one degree of freedom we will receive from the formula (35) after substitution of value $N_{H 2}$

$$
\eta_{1}=\eta_{A}^{2}=0.98
$$

The adaptive gearing moves with two degrees of freedom, if a output moment of resistance more than the input driving moment $M_{H_{2}}>M_{H 1}$.

In this case there is an internal relative motion of wheels 1 , 2, 3, 6, 5, 4 in the closed contour. A friction loss occurs not only in two rotational kinematic pairs $A$, unites the input and output drove with a rack, but also in a contour containing 4 rotational kinematic pairs (type $A$ ) and 4 higher kinematic 
pairs (type $C$ ). Therefore

$$
N_{H 2}=N_{H 1} \eta_{A}^{6} \eta_{C}^{4}
$$

Where $\eta_{C}=0.98$ - efficiency of the higher kinematic pair of type $C$.

Efficiency of all mechanism in a regime of motion with two degrees of freedom we will receive from the Eq. 35 after substitution of value $N_{H 2}$

$$
\eta_{2}=\eta_{A}^{6} \eta_{C}^{4}=0.87
$$

The adaptive gearing can have one more important regime of motion which occurs in case of the application of the greatest possible moment of resistance $M_{H_{2}} \geq M_{H 2 \max }$. In this case the output carrier is shut down at the input carrier continuing motion that is at the working propeller. Transfer continues to work in a condition with one degree of freedom. It is possible to name such regime of motion стоповым а regime. Stop the motion regime keeps readiness of transfer to begin motion at once after decrease of a output moment of resistance or at increase in power of an engine (without turning on of the propeller and repeated achievement of a regime of motion with the reduced moment of resistance, on what the additional time would be required). Stop the regime is necessary for work in extreme working conditions, for example, for the drive of military techniques. Therefore stop at the shut down output carrier it is impossible to consider a regime of motion of transfer as useless and having efficiency, equal to null.

Let's define efficiency стопового a motion regime at the motionless output carrier by formula

$$
\eta_{H 2}=1-\varphi \text {. }
$$

Where $\varphi$ - a loss factor.

Taking into account decrease of number of rotational kinematic pairs per unit transmission efficiency with the motionless output carrier can be defined by formula

$$
\eta_{H 2}=\eta_{A}^{5} \eta_{C}^{4}=0.88
$$

Loss factor at the motionless carrier

$$
\varphi_{H 2}=1-\eta_{H 2}=0.12
$$

However it is necessary to mean, that at the motionless carrier the intense high-speed regime of motion of wheels in a contour with increase in coefficient of friction at magnitude about $20 \%$ will occur. A friction loss accordingly will increase. A loss factor in the stop regime

$$
\varphi=1.2 \varphi_{H_{2}}=0.15 \text {. }
$$

Definitively efficiency стопового a motion regime

$$
\eta_{H 2}=1-\varphi=0.85 \text {. }
$$

\section{Practical Implementation}

An acting dummy gear stepless adjustable transfer is presented on fig. 7 .

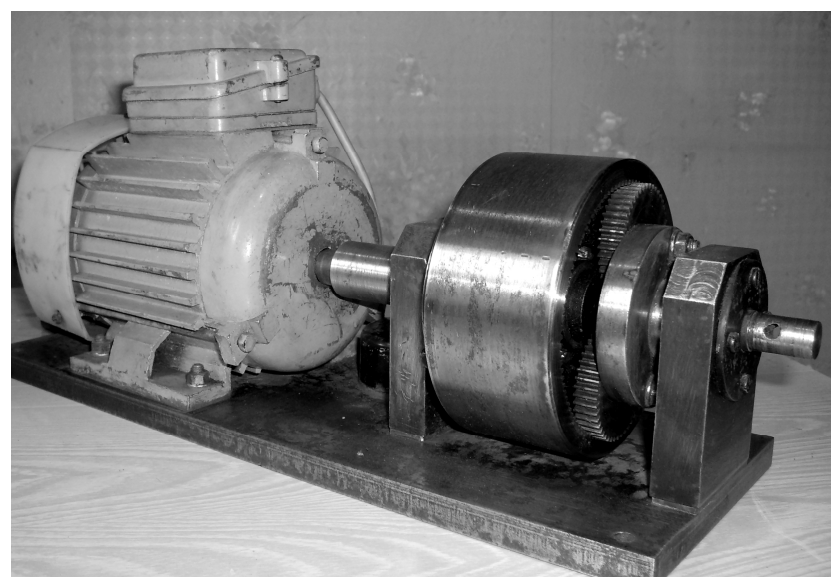

Fig. 3. The dummy gear stepless adjustable transfer

The dummy confirms presence of effect of force adaptation in a wheelwork with the closed contour.

\section{Conclusion}

The closed contour as a part of the kinematic chain leads to creation of contour mechanisms with basic new properties. In the kinematic chain with two degrees of freedom the closed contour provides definiteness of motion. Now it is proved, that the closed contour possesses property unknown earlier and as a part of the kinematic chain with one degree of freedom. New property of a contour consists that under act only an external impellent (in the absence of a resistance superposed force) the closed contour appears counterbalanced. On the basis of the executed researches it is possible to assert, that the closed contour always imposes additional communication on motion of links.

Thus, in the kinematic chain with two degrees of freedom the closed contour provides definiteness of motion as in a condition with two degrees of freedom (in motion operating conditions), and in a condition with one degree of freedom (at start-up). It allows considering the found regularity of the analysis of contour mechanisms as the classical theory applied to mechanical systems with ideal connections.

\section{References}

[1] Ivanov K.S., Dmitrieva N.A. Non reactive engine. The copyright certificate of the USSR №769157 from 7.09.1980. $10 \mathrm{p}$.

[2] Samuel J. Crockett. Shiftless, continuously-aligning transmission. Patent of USA 4,932,928, Cl. F16H 47/08, U.S. Cl. 475/51; 475/47.1990. 9 p.

[3] Harries John. Power transmission system comprising two sets of epicyclic gears. Patent of Great Britain GB2238090 (A). 1991. $11 \mathrm{p}$. 
[4] Ivanov K.S. Theoretical of a basis gear stepless adjustable transfer. The theory of mechanisms and machines. №2 (16). 2010. V. 8. SPb the state polytechnical university. St.-Petersburg. P. $36-48$.

[5] Ivanov K.S. The simplest automatic transfer box. WCE 2010. World Congress on Engineering 2010 (ICME) London, UK. 2010. - P. $1179-1184$.

[6] Ivanov K.S. Paradox of mechanics - a basis of creation CVT. Transactions of 2-d IFToMM Asian Conference on Mechanisms and Machines Science. Tokyo, Japan. November 7-10, 2012. P. $245-264$.

[7] Ivanov K.S., Yaroslavtseva E.K. Way of automatic and continuous change of the twisting moment and speed of rotation of a output shaft depending on resistance to motion and the device for its realization. Patent of Russia RU № 2398989. 10.09.2010. $10 \mathrm{p}$.

[8] Ivanov Konstantin S., Almaty, KAZ - Owner of the registered sample. The name - Device of automatic and continuous change of a twisting moment - and changes of a corrected speed of output shaft depending on a tractive resistance. The deed on registration of the registered sample № 202012101 273.1. Day of Registration 02.05.2012. The German patent and firm establishment. Federal Republic Germany. 2012. 12 p.

[9] Ivanov K.S., Yaroslavtseva E.K. Device of a transmission of energy with continuously variable transfer ratio (variants). Patent of Kazakhstan № 023907 from 23.02.2011. 16 p. 